\author{
Marquette University \\ e-Publications@Marquette
}

Electrical and Computer Engineering Faculty

Research and Publications

Electrical and Computer Engineering,

Department of

\title{
Fast Multi-Objective CMODE-Type Optimization of PM Machines Using Multicore Desktop Computers
}

\author{
Alireza Fatemi \\ Marquette University \\ Dan M. Ionel \\ University of Wisconsin - Milwaukee \\ Nabeel Demerdash \\ Marquette University, nabeel.demerdash@marquette.edu \\ Thomas W. Nehl \\ General Motors
}

Follow this and additional works at: https://epublications.marquette.edu/electric_fac

Part of the Computer Engineering Commons, and the Electrical and Computer Engineering Commons

\section{Recommended Citation}

Fatemi, Alireza; Ionel, Dan M.; Demerdash, Nabeel; and Nehl, Thomas W., "Fast Multi-Objective CMODEType Optimization of PM Machines Using Multicore Desktop Computers" (2016). Electrical and Computer Engineering Faculty Research and Publications. 210.

https://epublications.marquette.edu/electric_fac/210 
Marquette University

e-Publications@Marquette

\title{
Electrical and Computer Engineering Faculty Research and Publications/College of Engineering
}

This paper is NOT THE PUBLISHED VERSION.

Access the published version at the link in the citation below.

IEEE Transactions on Industry Applications, Vol. 52, No. 4 (July-August 2016): 2941-2950. DOI. This article is (C) The Institute of Electrical and Electronics Engineers and permission has been granted for this version to appear in e-Publications@Marquette. The Institute of Electrical and Electronics Engineers does not grant permission for this article to be further copied/distributed or hosted elsewhere without the express permission from The Institute of Electrical and Electronics Engineers.

\section{Fast Multi-Objective CMODE-Type Optimization of PM Machines Using Multicore Desktop Computers}

\author{
Alireza Fatemi \\ Department of Electrical and Computer Engineering, Marquette University, Milwaukee, WI \\ Dan M. Ionel \\ Department of Electrical and Computer Engineering, University of Kentucky, Lexington, KY \\ Nabeel A. O. Demerdash \\ Department of Electrical and Computer Engineering, Marquette University, Milwaukee, WI \\ Thomas W. Nehl \\ General Motors Global Research and Development, Warren, MI
}




\begin{abstract}
:
Large-scale design optimization of electric machines is oftentimes practiced to achieve a set of objectives, such as the minimization of cost and power loss, under a set of constraints, such as maximum permissible torque ripple. Accordingly, the design optimization of electric machines can be regarded as a constrained optimization problem (COP). Evolutionary algorithms (EAs) used in the design optimization of electric machines including differential evolution (DE), which has received considerable attention during recent years, are unconstrained optimization methods that need additional mechanisms to handle COPs. In this paper, a new optimization algorithm that features combined multi-objective optimization with differential evolution (CMODE) has been developed and implemented in the design optimization of electric machines. A thorough comparison is conducted between the two counterpart optimization algorithms, CMODE and DE, to demonstrate CMODE's superiority in terms of convergence rate, diversity and high definition of the resulting Pareto fronts, and its more effective constraint handling. More importantly, CMODE requires a lesser number of simultaneous processing units which makes its implementation best suited for state-of-the-art desktop computers reducing the need for high-performance computing systems and associated software licenses.
\end{abstract}

\title{
SECTION I. Introduction
}

Large-scale design optimization techniques have become a well-established practice for designing highperformance electric machines [1][2][3][4][5][6][7]-[8]. In these techniques, the parametrized cross section of a subject machine is refined to improve certain performance metrics with respect to the application requirements. Cost, power loss, torque density, torque ripple, power factor, and degrees of demagnetization of rotor permanent magnets (PMs) in PM machines are common performance metrics which constitute the set of objectives and constraints in any optimization problem.

In principle, a large-scale model-based design optimization process consists of two independent segments: 1) the machine model for computation of performance metrics and 2) the optimizer for finding the globally optimal design solutions. Regarding the machine model, both analytical [1][2][3][4] and finite-element (FE) [5][6][7]-[8] methods are commonly used in a large-scale design optimization process, with the latter receiving more attention during recent years owing to the everincreasing processing power of modern computers. Concerning the optimizer, either a deterministic or stochastic search algorithm can be used.

Efficient utilization of computational resources is imperative when the performance evaluation of the design candidates is computationally intensive [9], as in the case of the FE models. Two areas of opportunity exist under each segment of the optimization process that can serve this purpose. On the performance evaluation side, computationally efficient-finite-element analysis (CE-FEA) has been recently introduced [5], [10] for fast- and high-fidelity simulation of PM machines. On the optimizer side, the differential evolution (DE) [11] has received extensive attention as a reliable and fast stochastic search algorithm [12], [13]. The DE is thought to have a better performance in comparison with other stochastic optimizers in electric machinery design problems [11], [13]. It has been coupled to the CE-FEA for optimization of several types of PM motors with various sets of objectives and constraints [5][6][7]-[8], [14]. 
Although DE has proved effective in the design optimization of electric machines [15], similar to other evolutionary algorithms (EAs), it has not been developed for handling constrained optimization problems (COPs) [16], [17], which is the case in design of electric machines [15], [18]. Popular constraint handling mechanisms include penalty function methods, methods based on preference of feasible solutions, and multi-objective optimization techniques [16], [19]. In the latter, COPs are converted to unconstrained multi-objective optimization problems where minimization of the so-called degree of constraint violation is designated as an additional objective.

In this paper, a recently developed combined multi-objective optimization with differential evolution (CMODE) [16], [20] is adapted for the design optimization of electric machines with application to three IPM motors with distributed and concentrated stator windings. The same design problem is performed using the standard DE to compare the outcomes with those obtained from the CMODE approach. It is demonstrated here that CMODE is superior to DE in terms of convergence rate and constraint handling in all the three example motor configurations. Furthermore, CMODE requires a lesser number of simultaneous function evaluations which makes it an attractive solution for implementation of the design optimization on a state-of-the-art desktop computer with a limited number of processors, thus reducing the need for high-performance computing (HPC) facilities and associated software licenses. In Section II, the essence of the two optimization algorithms and their similarities and differences are discussed. The benchmark studies are explained in Section III followed by the optimization results and the comparison between the two optimization algorithms in Section IV. The conclusion of this paper is given in Section $\mathrm{V}$.

\section{SECTION II. CMODE Versus DE in the Design Optimization of Electric}

\section{Machines}

The flowchart of steps of the two counterpart search algorithms, DE and CMODE, applied to the design optimization of electric machines is shown in Figs. 1 and 2, respectively. In both cases, a preliminary design is obtained analytically in reference to the application requirements and specifications [21]. This initial design is subsequently parametrized and the geometric design variables and constants are specified in the initialization stage. Preparation of a well-defined parameterized model, which on one hand is flexible for the exploration of the entire design space, and on the other hand is restrained to avoid geometric conflicts between various components of the machine cross section, is a nontrivial demanding task. 


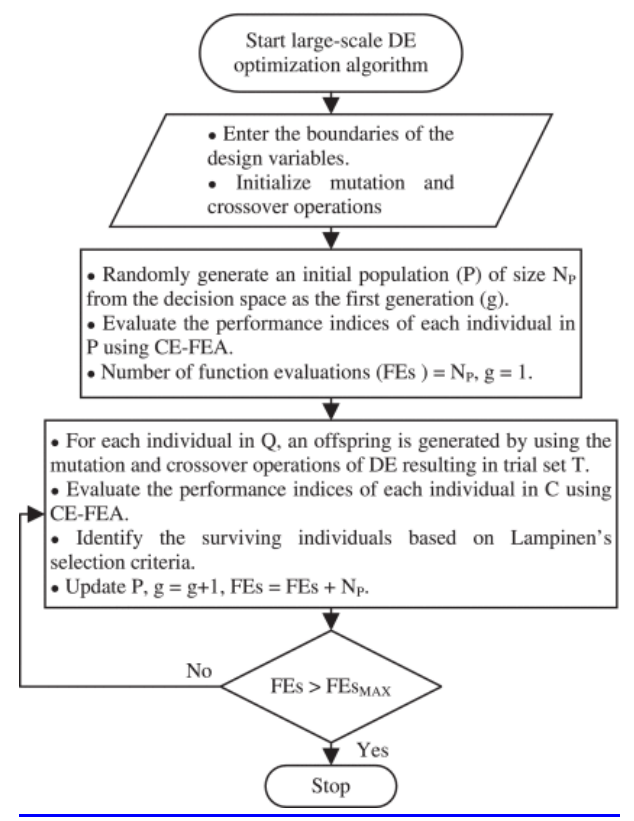

Fig. 1. Flowchart of the steps of the DE optimization algorithm.

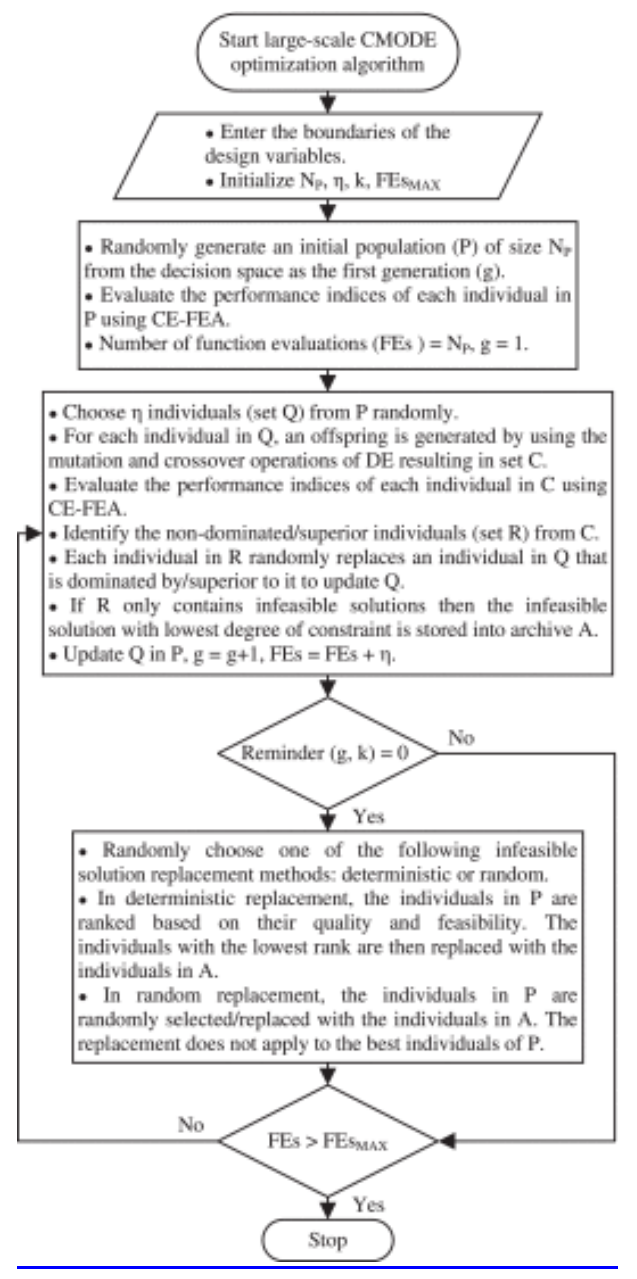

Fig. 2. Flowchart of the steps of the CMODE optimization algorithm. 
The optimization process involves performance assessment of the resulting design candidates. For this purpose, the CE-FEA approach [5], [10], which accommodates the complex geometry of the machine structure, and incorporates the actual nonlinear nature of the magnetic core, is utilized.

Apart from using the same parameterized model and the same technique for performance evaluation of the design candidates, here the CE-FEA method, the optimization procedure differs for the CMODE and the DE algorithms in the following manner.

1. Unlike DE in which, as a standard $E A$, all the population members are used to generate the offspring population, in CMODE, only a portion of the total individuals, denoted by $\eta$, are chosen for this purpose. This renders CMODE a steady-state EA where the first randomly generated population has a large number of members, a fraction of which, set $Q$, are being constantly updated throughout the optimization process. Consequently, CMODE performs a fraction of simultaneous function evaluations (FEs) contrary to what takes place in DE. Here, performing FEs means applying the CE-FEA approach in solving the electromagnetic field in the design candidate machines. Typical numbers recommended for DE are 60 generations each consisting of 80 members [7], [8], in contrast with an initial population of 180 members followed by 400 generations each consisting of eight members recommended for CMODE [16]. Lesser number of simultaneous FEs, here 8 versus 60, makes CMODE's implementation best suited for state-of-the-art desktop computers.

2. The selection procedure for determination of the surviving candidates in CMODE is based on the identification of superior individuals in the offspring population, set $C$, and having them replace the dominated individuals in the parent population, set $Q$. Therefore, in comparison to $D E$, there is an additional round of competition in CMODE. The first round is between all the individuals in the offspring population, and the second round is between the winners of the first round, set $R$, and the individuals in the parent population. Nevertheless, CMODE still benefits from the mutation and crossover operations of DE [16], which produces the trial and ultimately the offspring populations of consecutive generations.

3. In $\mathrm{CMODE}$, an additional variable defined as the degree of constraint violation is introduced into the objective function to be minimized with other objectives. Let $\vec{x}$ be the design vector, and $f(\vec{x})$ be the initial objective of the optimization problem subjected to a set of q inequality constraints, $g_{j}(\vec{x})$, and $(m-q)$ equality constraints, $h_{j}(\vec{x})$. The final objective of the optimization process would be the minimization of $\mathbf{f}(\overrightarrow{\mathbf{x}})=(f(\vec{x}), G(\vec{x}))$, where $G(\vec{x})$ is the degree of constraint violation given as follows [16]:

$$
G(\vec{x})=\sum_{j=1}^{m} G_{j}(\vec{x})
$$

where 


$$
G_{j}(\vec{x})=\left\{\begin{array}{c}
\max \left\{0, g_{j}(\vec{x})\right\}, 1 \leq j \leq q \\
\max \left\{0,\left|h_{j}(\vec{x})\right|-\delta\right\}, q+1 \leq j \leq m .
\end{array}\right.
$$

4. Finally, according to Fig. 2, CMODE features an infeasible solution replacement mechanism in which after a certain number of generations, denoted by $k$, an archive consisting of individuals that violate the constraints, $A$, replaces the individuals in the main population, $P$, either through a deterministic or random procedure. This mechanism adds to the diversity of the overall optimization problem to accommodate convergence to the globally optimal solutions.

\section{SECTION III. Benchmark Study: Application to IPM Motors}

\section{A. Parametrized FE Model}

To compare the merits of the two counterpart stochastic search algorithms, three IPM motor configurations with distinctive rotor and stator features and under different loading conditions have been investigated.

1. A fan-cooled 48-slot, eight-pole motor with single-layer $\mathrm{v}$-shaped magnets and single-layer distributed winding configuration. This design will be referred to as the 48S8P-a design for brevity.

2. A liquid-cooled 48-slot, eight-pole motor with double-layer v-shaped magnets and single-layer distributed winding configuration, which will be referred to as the 48S8P-b design.

3. A naturally cooled 12-slot, 10-pole motor with single-layer v-shaped magnets and double-layer concentrated winding configuration, called hereafter the 12S10P design.

The current density of the stator winding can be adjusted to account for the ampere-loading of the machine. Typical current density ranges are provided in Table I [21]. Here, 22, 8, and $4 \mathrm{~A} / \mathrm{mm}^{2}$ are assumed for liquid-, fan-, and naturally cooled machines, respectively. The variety introduced to the selected machine configurations and their electrical loadings provides the basis for a rigorous comparison between the two search algorithms.

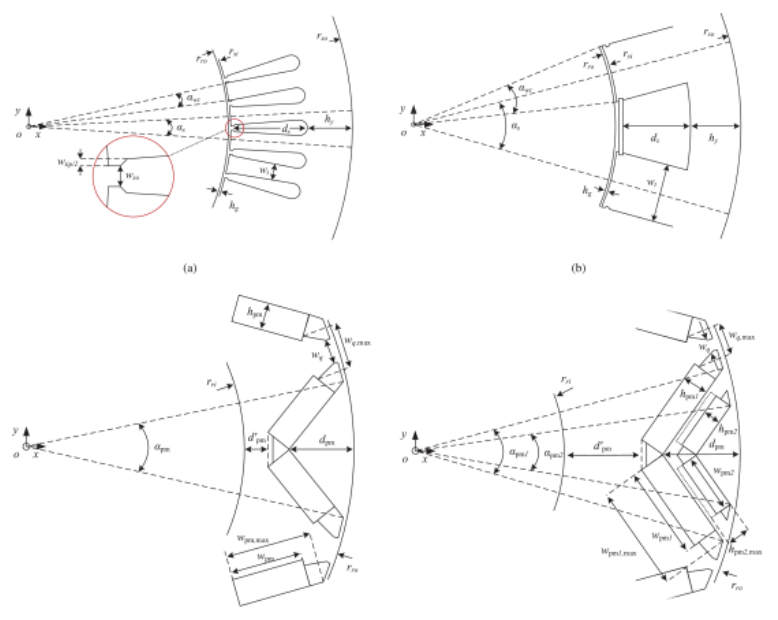

Fig. 3. Parameterized structures used for constructing the example IPM motors. (a) 48-slot stator. (b) 12-slot stator. (c) Single-layer rotor PM layout. (d) Double-layer rotor PM layout. 
TABLE I Typical Current Density Ranges for Different Cooling Systems

\begin{tabular}{|l|l|l|l|}
\hline Cooling & Natural & Fan & Liquid \\
\hline Current density $\left(\mathrm{A} / \mathrm{mm}^{2}\right)$ & $1.5-5$ & $5-10$ & $10-30$ \\
\hline
\end{tabular}

To construct the FE model of the example machines, the parametrized stator and rotor structures shown in Fig. 3 are utilized. The independent design variables defined based on these parametrized models are listed in Table II. Some of these design variables are rationalized according to Table II so as to avoid geometric conflicts between the structures of various components of the motor. The parameterized FE model of the 48S8P-a motor comprises a total of ten independent design variables, five residing in the rotor, four residing in the stator, in addition to the air-gap height. The 48S8P-b motor has two additional independent variables introduced to the rotor geometry to accommodate the double-layer PMs. Meanwhile, the open-slot 12S10P motor has one less independent design variable because of its open slot structure. The design variables are confined by upper and lower bounds, also listed in Table II and depicted in Fig. 3 for some of the variables in a typical design, either to prevent the unintended intersection of various boundary surfaces of machine components, or to address mechanical constraints, e.g., minimum air-gap height or the yield stress for the rotor bridges [22]. For all the three machines, the stator outer diameter is fixed to $260 \mathrm{~mm}$. The shaft diameter is equal to $111 \mathrm{~mm}$ and $74 \mathrm{~mm}$ for the single-layer and double-layer rotor magnet configurations, respectively. The parameterized geometry together with the introduced bounds allow the model to be flexible in exploring the entire design space to find the globally optimized design candidates.

TABLE II Independent Design Variables and Their Upper and Lower Bounds, See Fig. 3

\begin{tabular}{|l|l|l|l|l|l|l|l|}
\hline Parameters & Description & 48S8P-a & & 48S8P-b & & 12S10P & \\
\hline & & \multicolumn{1}{|c|}{$x_{i, \min }$} & \multicolumn{1}{|c|}{$x_{i, \max }$} & \multicolumn{1}{|c|}{$x_{i, \min }$} & $x_{i, \max }$ & $x_{i, \min }$ & $x_{i, \max }$ \\
\hline$k_{\mathrm{si}}$ & $r_{\mathrm{si}} / r_{\mathrm{so}}$ & 0.6 & 0.7 & 0.6 & 0.7 & 0.6 & 0.7 \\
\hline$h_{g}(\mathrm{~mm})$ & Fig. 3 & 0.7 & 2.5 & 0.7 & 2.5 & 0.7 & 2.5 \\
\hline$k_{w_{t}}$ & $\alpha_{w_{t}} / \alpha_{w_{s}}$ & 0.35 & 0.75 & 0.35 & 0.75 & 0.35 & 0.75 \\
\hline$k_{w_{t t}}$ & $w_{\text {tip }} /\left(w_{\text {so }}+w_{\text {tip }}\right)$ & 0.3 & 0.8 & 0.3 & 0.8 & NA & NA \\
\hline$k_{d_{\mathrm{pm}}}$ & $d_{\mathrm{pm}} /\left(d_{\mathrm{pm}}+d_{\mathrm{pm}}^{\prime}\right)$ & 0.25 & 0.50 & 0.25 & 0.50 & 0.15 & 0.65 \\
\hline$k_{w_{\mathrm{pm}}}$ & $w_{\mathrm{pm}} / w_{\mathrm{pm} \text { max }}$ & 0.80 & 0.93 & 0.80 & 0.93 & 0.76 & 0.94 \\
\hline$k_{w_{q}}$ & $w_{q} / w_{q, \max }$ & 0.5 & 0.9 & 0.65 & 0.90 & 0.3 & 0.8 \\
\hline$h_{\mathrm{pm}}(\mathrm{mm})$ & Fig. 3 & 3.8 & 9.0 & 3.8 & 9.0 & 2.5 & 8.0 \\
\hline$\alpha_{\mathrm{pm}}(\mathrm{deg})$. & Fig. 3 & 20 & 32 & 20 & 32 & 19 & 26 \\
\hline$h_{y}(\mathrm{~mm})$ & Fig. 3 & 13 & 25 & 13 & 25 & 13 & 25 \\
\hline$k_{\alpha_{\mathrm{pm}}}$ & $\alpha_{\mathrm{pm} 1} / \alpha_{\mathrm{pm} 2}$ & NA & NA & 0.3 & 0.8 & NA & NA \\
\hline$k_{h_{\mathrm{pm}}}$ & $h_{\mathrm{pm} 2} / h_{\mathrm{pm} 2 \text { max }}$ & NA & NA & 0.4 & 0.8 & NA & NA \\
\hline
\end{tabular}

The CE-FEA method is utilized for fast- and high-fidelity calculation of the machine performance metrics [5], [10]. According to this method, if a balanced three-phase sinusoidal current excitation is 
assumed, the electric symmetry in the stator geometry can be used to map the tangential $f_{T}$ and radial $f_{R}$ field values between sister elements throughout the stator periodicity span, see Fig. 4 for a typical illustration in the 48-slot stator, as given by

$$
f_{R, T}\left(t+\frac{k \alpha_{s} P}{2 \omega}, r, \theta\right)=f_{R, T}\left(t, r, \theta+k \alpha_{s}\right)
$$

(2)

where $k$ is the index that depends on the slot-pole combination and winding layout, and $\alpha_{s}$ is the slot pitch in mechanical measure.

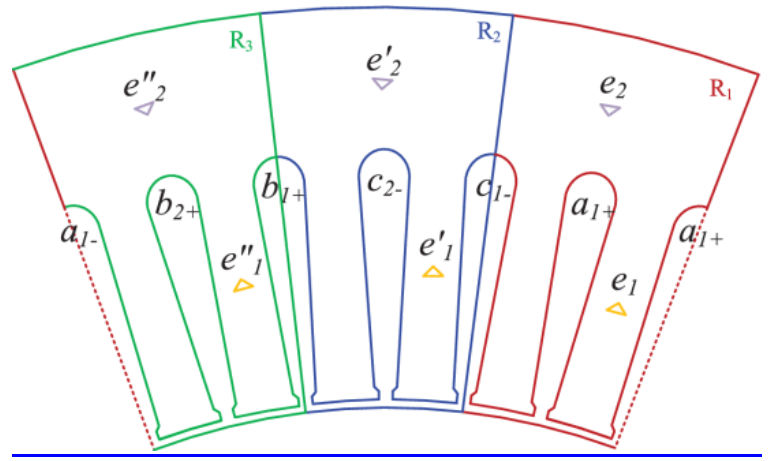

Fig. 4. Field values between typical sister elements such as $e_{1}, e_{1}^{\prime}$, and $e_{1}^{\prime \prime}$ or $e_{2}, e_{2}^{\prime}$, and $e_{2}^{\prime \prime}$ are mapped in the CE-FEA method.

Using the CE-FEA allows reconstruction of the entire field waveforms through multiple snapshots of magnetostatic FE solutions over a time span corresponding to 60 electrical deg. The CE-FEA can be up to two orders of magnitude faster compared to the full-fledged time-stepping transient FE solutions [5], [10]. It has been demonstrated to be effective in large-scale design optimizations of PM machines with various rotor layouts and stator winding configurations, including experimental verifications [5][6][7]-[8], [14].

\section{B. Optimization Fitness Functions}

Since the purpose of the optimization is a comparative study between the search algorithms, the fitness function of the optimization problem can be chosen arbitrarily. In a practical case, the performance metrics of interest can be the machine's active material cost, power losses, torque ripple, and the degree of demagnetization of the PMs.

1. Active material cost $A M C$ is given by

$$
\mathrm{AMC}=24 \cdot m_{\mathrm{PM}}+3 \cdot m_{\text {copper }}+m_{\text {steel }}
$$

where the mass $m$ is in $\mathrm{kg}$ and the steel cost is considered as the one-unit reference in this normalized/per-unit formulation. 
2. The power losses consist of copper losses in windings, and the stator core losses including hysteresis and eddy current losses. The frequency domain core loss model introduced in [23] is adopted for calculation of the stator core losses. The PM losses are considered in the 12S10P machine using the method introduced in [24].

3. As mentioned previously, the stator outer diameter and the rotor inner diameter are held constant. Following the calculation of the average torque per unit length, using CE-FEA for the rated current density, the stack-length of the designs is adjusted accordingly to produce the desired average $300 \mathrm{Nm}$ torque at $1500 \mathrm{rev} / \mathrm{min}$. The torque ripple is subsequently determined from the torque profile over a full fundamental ac cycle.

4. The degree of PM demagnetization is characterized by the minimum flux density in the rotor PMs over a complete ac cycle. Demagnetization is considered at the magnet piece level.

The fitness functions of the optimization problem can now be built upon the discussed performance metrics. Two different scenarios of objectives and constraints are pursued for a rigorous comparison between the CMODE and the DE algorithms. In both scenarios, two objectives subjected to two constraints are considered. The first scenario consists of the following two objectives and constraints.

1. Objectives: (a) minimization of $A M C$ and (b) minimization of power losses.

2. Constraints: (a) torque ripple less than $15 \%$ and (b) PM demagnetization less than $70 \%$. The second scenario is designated as follows.

1. Objectives: (a) minimization of torque ripple and (b) minimization of power losses.

2. Constraints: (a) axial stack length less than 200, 70, and $400 \mathrm{~mm}$ for the 48S8P-a, 48S8P-b, and 12S10P machines, respectively, and (b) PM demagnetization less than $70 \%$.

\section{SECTION IV. Comparative Study of the Results}

Twelve runs of large-scale design optimizations were carried out on a desktop workstation using eight simultaneous processing units and eight ANSYS Maxwell distributed solvers. The machines were optimized for the two aforementioned fitness functions, using either the DE or the CMODE as the stochastic optimizer. The DE consists of 40 generations, each of 80 members. The CMODE starts with an initial generation of 180 members and proceeds with 378 eight-member generations. The number of members in each generation is recommended by [8], [13] for DE and [16] for CMODE. The overall number of design evaluations is approximately equal, 3200 designs in DE versus 3204 designs in CMODE.

In Figs. 5 and 6, the progress of the optimization process in terms of the conflicting objectives, which are normalized independently for each example machine, is illustrated for the two sets of fitness functions. The number of the function evaluations, i.e., the sequence of the candidate designs, is color coded to provide an indication of the convergence of the design space to the Pareto front vicinity. Furthermore, the designs are differentiated based on their feasibility to provide an indication of the effectiveness of the constraint handling in the two optimization algorithms. It can be seen in these figures that both DE and CMODE successfully converge to the same optimal neighborhood in the 
design space. However, the concentration of the feasible design candidates in the Pareto front vicinity is larger for CMODE, resulting in a better-defined Pareto front with a lesser number of design evaluations as opposed to DE, also see Fig. 7. In addition, the color code in Figs. 5 and 6 suggests that the convergence to the Pareto front solutions is faster for CMODE.
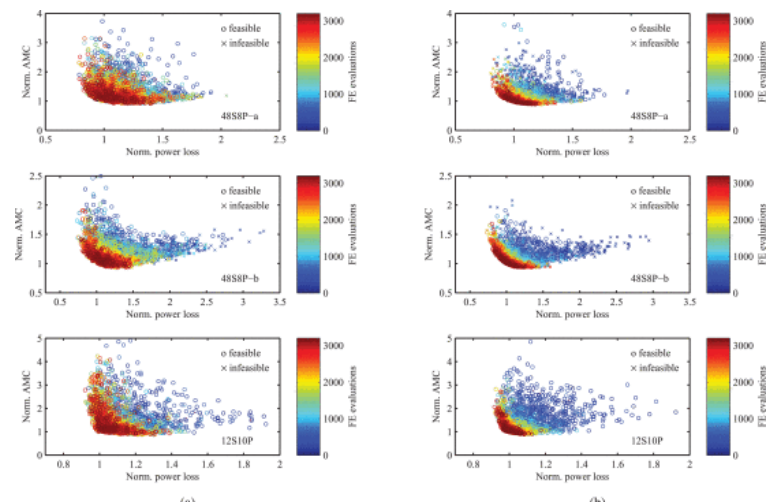

Fig. 5. Evolution of the optimization process using (a) DE and (b) CMODE for the three case-study motors under the first scenario.
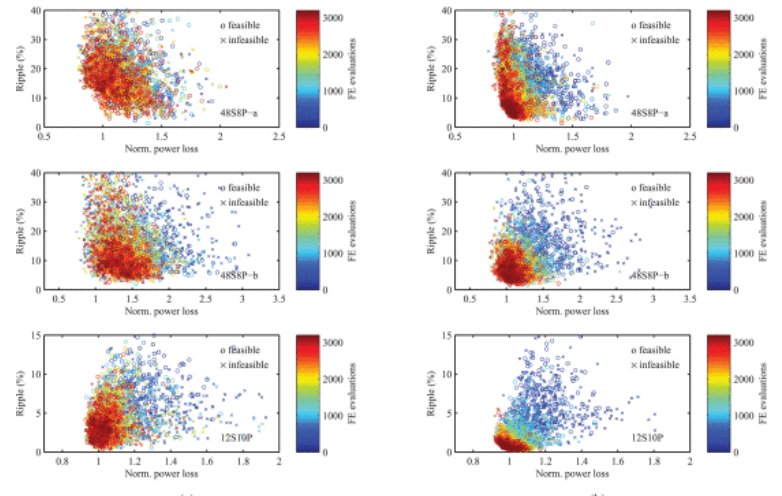

Fig. 6. Evolution of the optimization process using (a) DE and (b) CMODE for the three case-study motors under the second scenario.
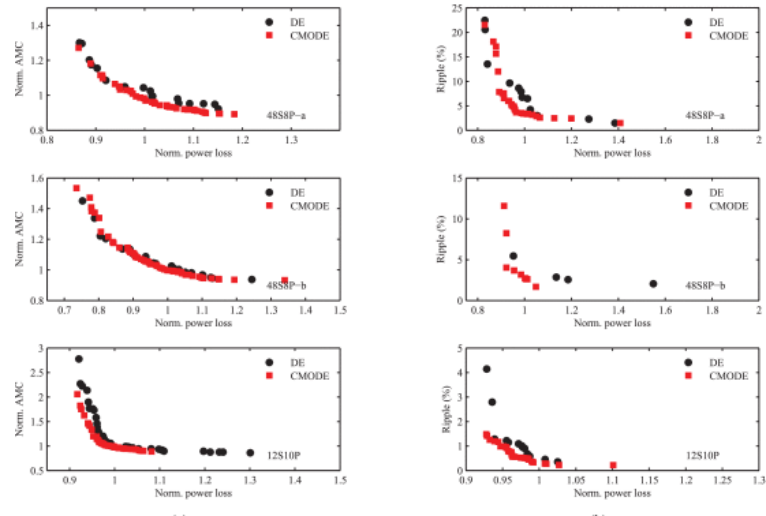

Fig. 7. Feasible Pareto optimal designs of the two optimization algorithms. (a) Scenario 1 and (b) scenario 2 of the fitness functions.

A comparison between Figs. 5 and 6 reveals that the realization of the objectives and constraints is more difficult in the second scenario of the fitness functions. Nonetheless, CMODE is still superior to DE as shown in Figs. 6(a), (b) and 7(b). 
To further discern the difference between the convergence rates of the two search algorithms, an auxiliary variable is defined as the normalized product of the two objectives in the feasible design candidates. The decay of this quantity over simulation time can serve as an indication of the optimization progress. The mean of this quantity per each generation of optimization is shown in Fig. 8 . The two previous observations regarding the faster convergence rate of CMODE, and the denser concentration of the feasible design solutions in the vicinity of the Pareto front, are distinctly verified in these figures. As can be seen in Fig. 8, the duration and the convergence rate of the two optimization algorithms are very much dependent on the problem definitions. Yet, compared to DE, CMODE reaches steady state at least twice as fast in all the 12 different case studies. The optimizations can be continued in steady state until the Pareto front acquires a well-defined profile. Since the majority of the simulation time is spent to solve the FE-based models, as opposed to the fraction of seconds spent by the optimization search algorithms, the total duration of the optimization procedure is equal for the two algorithms, given that the number of FEs is the same. However, the CMODE algorithm is able to produce a large number of optimal designs in contrary to the DE algorithm, for which the simulation needs to be continued.
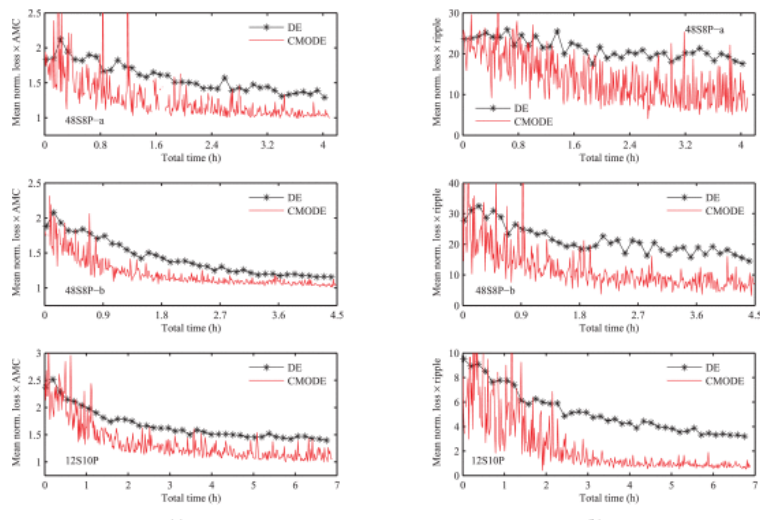

Fig. 8. Convergence of the feasible design candidates for the three case-study motors in terms of (a) loss $\times$ AMC for scenario 1 and (b) loss $X$ ripple for scenario 2 .

As shown in Fig. 7, and according to Table III, CMODE also provides a larger number of optimal solutions in the immediate vicinity of the Pareto front, resulting in CMODE's higher definition and better diversity of Pareto front solutions. To quantitatively compare the Pareto fronts of the two optimization algorithms, the hypervolume indicator [25] is calculated. The hypervolume measures the dominance of the Pareto front solutions with respect to a reference point in the decision space [26]. Here, it is calculated with respect to the maximum objective values for each set of Pareto front solutions. The normalized hypervolumes of the two optimization algorithms in Fig. 9 clearly indicate the persistent superiority of CMODE in terms of diversity and quality of generated Pareto fronts. 


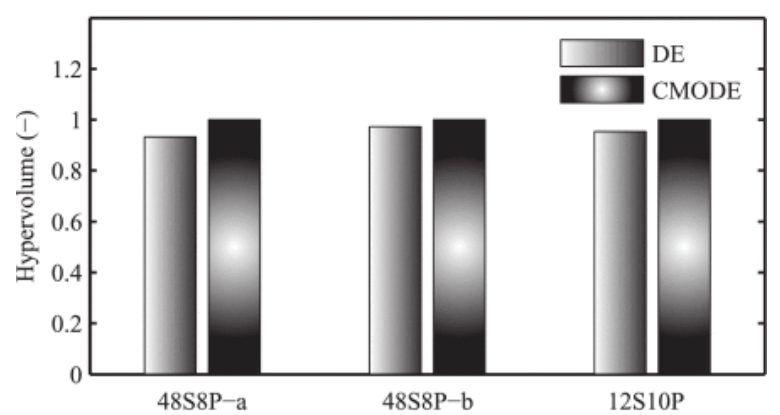

(a)

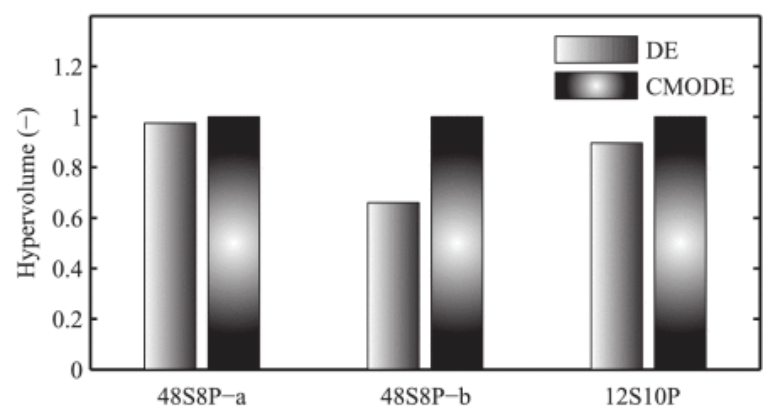

(b)

Fig. 9. Normalized hypervolumes of the Pareto fronts generated by DE and CMODE. (a) Scenario 1 and (b) scenario 2 of fitness functions.

TABLE III Number of the Feasible Pareto Optimal Designs for the Two Scenarios of Fitness Functions

\begin{tabular}{|l|l|l|l|l|}
\hline Machine & Scenario I & & Scenario 2 & \\
\hline & DE & CMODE & DE & CMODE \\
\hline 48S8P-a & 17 & 42 & 12 & 28 \\
\hline 48S8P-b & 24 & 52 & 4 & 10 \\
\hline 12S IOP & 34 & 41 & 13 & 30 \\
\hline
\end{tabular}

The two search algorithms are also compared in terms of their constraint handling capability in Table IV. It can be seen that the overall number of the feasible design candidates, which pass the two constraints imposed either on the torque ripple and on the PM demagnetization in the first scenario, or on stack length and PM demagnetization in the second scenario is higher in CMODE. The effective constraint handling of the CMODE algorithm, in addition to the denser designs in its Pareto front vicinity, translates into its superior computational efficiency when compared to DE.

TABLE IV Number of Total Feasible Design Candidates for the Two Scenarios of Fitness Functions

\begin{tabular}{|l|l|l|l|l|}
\hline Machine & Scenario I & & Scenario 2 & \\
\hline & DE & CMODE & DE & CMODE \\
\hline 48S8P-a & 1136 & 1189 & 1780 & 2306 \\
\hline 48S8P-b & 1500 & 2256 & 1438 & 1731 \\
\hline 12S10P & 3162 & 3199 & 1387 & 1849 \\
\hline
\end{tabular}


Typical cross sections of the optimal design solutions and their field plots for each scenario are provided in Fig. 10.

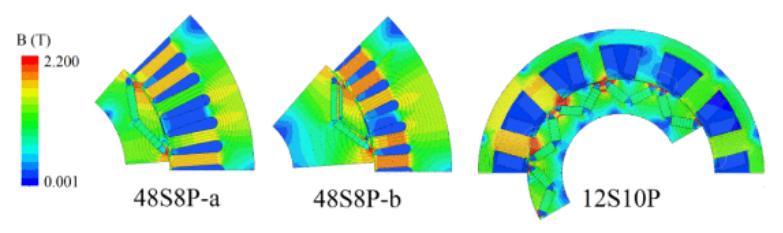

(a)

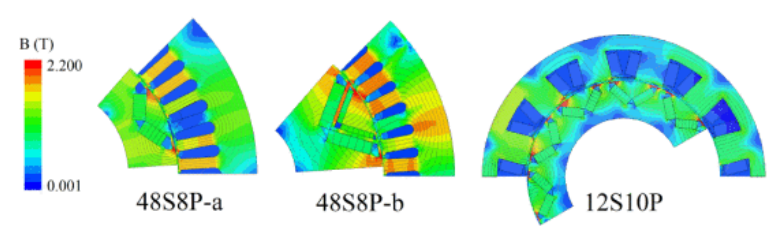

(b)

Fig. 10. Typical optimized cross sections and the field plots of the studied motors under (a) scenario 1 and (b) scenario 2 of the fitness functions.

Because of the sequential processing of generations in synchronous EAs such as the above implementations of DE and CMODE, the optimum number of distributed solvers is equal to the number of members in each generation. It should be emphasized that in the foregoing comparison between $D E$ and $C M O D E$, eight distributed solvers corresponding to the number of members in each generation of CMODE were utilized. This number of parallel solvers can be readily implemented on a single desktop computer with limited processing power and with minimum number of software licenses, e.g., eight processing cores and licenses in this investigation. The CMODE algorithm will maintain the discussed superiorities in comparison to DE if a lesser number of distributed solvers were to be used, which is usually the case for smaller motor design companies/groups. However, for implementation on a large number of distributed solvers, such as in HPC systems, the speed of simulations in DE as a standard EA will linearly increase up to the number of members in each generation, whereas the additional computational resources cannot be fully utilized in the CMODE algorithm as a steady-state EA, although CMODE still benefits from a faster convergence rate per generation and a more effective constraint handling mechanism. This highlights the importance of choosing the proper optimization algorithm in reference to the available computational resources, a topic that has been the focus of this paper by introducing CMODE for implementation on desktop computers. Under these premises, the performance of CMODE is expected to surpass other standard population-based multi-objective EAs which have been compared with DE in [9], [11], [13], and [16].

\section{SECTION V. Conclusion}

A new CMODE-type algorithm has been developed for design optimization of electric machines with limited computational resources. In the case study, IPM motors with distinctive stator winding configurations, rotor layouts, and electrical loadings, CMODE consistently demonstrated a faster convergence, at least twice as fast as the convergence rate of $D E$, a higher definition of Pareto front, and a better constraint handling in comparison with DE. These can be attributed to the distinctive population evolution model of CMODE, and its effective constraint handling method, in which the 
degree of constraint violation is being minimized simultaneously with other objectives of the optimization problem.

In design problems with more than one constraint, such as the case study examples in this paper, it might be required to introduce particular weights in the summation operation given in the definition of the degree of constraint violation. These weights have been designated so as to make the violations comparable between all the constraints. This can be accomplished, e.g., by normalizing such violations with respect to their expected range of variations. The assignment of the weights needs expert knowledge and particular attention in the implementation of CMODE for electric machinery design problems.

CMODE's fast convergence and fewer number of simultaneous function evaluations make it best suited for implementation in a state-of-the-art multiprocessor desktop computer with a lesser number of software licenses, as opposed to the high-end HPC systems.

\section{ACKNOWLEDGMENT}

The authors would like to thank ANSYS Inc. and Motor Design Limited for the software support.

\section{References}

1. R. Ramarathnam, B. G. Desai and V. S. Rao, "A comparative study of minimization techniques for optimization of induction motor design", IEEE Trans. Power App. Syst., vol. PAS-92, no. 5, pp. 1448-1454, Sep. 1973.

2. N. Bianchi and S. Bolognani, "Brushless DC motor design: An optimisation procedure based on genetic algorithms", Proc. 8th Int. Elect. Mach. Drives, pp. 16-20, Sep. 1997.

3. D.-J. Sim, D.-H. Cho, J.-S. Chun, H.-K. Jung and T.-K. Chung, "Efficiency optimization of interior permanent magnet synchronous motor using genetic algorithms", IEEE Trans. Magn., vol. 33, no. 2, pp. 1880-1883, Mar. 1997.

4. B. N. Cassimere and S. D. Sudhoff, "Population-based design of surface-mounted permanent-magnet synchronous machines", IEEE Trans. Energy Convers., vol. 24, no. 2, pp. 338-346, Jun. 2009.

5. G. Sizov, P. Zhang, D. M. Ionel, N. A. O. Demerdash and M. Rosu, "Automated multi-objective design optimization of PM AC machines using computationally efficient FEA and differential evolution", IEEE Trans. Ind. Appl., vol. 49, no. 5, pp. 2086-2096, Sep./Oct. 2013.

6. G. Y. Sizov, D. M. Ionel and N. A. O. Demerdash, "Modeling and parametric design of permanentmagnet AC machines using computationally efficient finite-element analysis", IEEE Trans. Ind. Electron., vol. 59, no. 6, pp. 2403-2413, Jun. 2012.

7. P. Zhang et al., "Multi-objective tradeoffs in the design optimization of a brushless permanent magnet machine with fractional-slot concentrated windings", Proc. IEEE Energy Convers. Congr. Expo. (ECCE), pp. 2842-2849, Sep. 2013.

8. P. Zhang, D. lonel and N. Demerdash, "Saliency ratio and power factor of IPM motors optimally designed for high efficiency and low cost objectives", Proc. IEEE Energy Convers. Congr. Expo. (ECCE), pp. 3541-3547, Sep. 2014.

9. G. Bramerdorfer, A. C. Zavoianu, S. Silber, E. Lughofer and W. Amrhein, "Speed improvements for the optimization of electrical machines-A survey", Proc. IEEE Int. Elect. Mach. Drives Conf. (IEMDC), pp. 1748-1754, May 2015. 
10. D. M. Ionel and M. Popescu, "Finite element surrogate model for electric machines with revolving field-Application to IPM motors", Proc. IEEE Energy Convers. Congr. Expo. (ECCE), pp. 178-186, Sep. 2009.

11. K. V. Price, R. M. Storn and J. A. Lampinen, Differential Evolution-A Practical Approach to Global Optimization, Heidelberg, Germany:Springer-Verlag, Jan. 2006.

12. D. Žarko and S. Stipetić, "Criteria for optimal design of interior permanent magnet motor series", Proc. 20th Int. Elect. Mach. (ICEM), pp. 1242-1249, Sep. 2012.

13. Y. Duan and D. M. Ionel, "A review of recent developments in electrical machine design optimization methods with a permanent magnet synchronous motor benchmark study", IEEE Trans. Ind. Appl., vol. 49, no. 3, pp. 1268-1275, May/Jun. 2013.

14. Y. Duan and D. M. Ionel, "Nonlinear scaling rules for brushless PM synchronous machines based on optimal design studies for a wide range of power ratings", IEEE Trans. Ind. Appl., vol. 50, no. 2, pp. 1044-1052, Mar./Apr. 2014.

15. W. Ouyang, D. Zarko and T. A. Lipo, "Permanent magnet machine design practice and optimization", Proc. 41st IEEE IAS Annu. Meeting Record Ind. Appl., vol. 4, pp. 1905-1911, 2006.

16. Y. Wang and Z. Cai, "Combining multiobjective optimization with differential evolution to solve constrained optimization problems", IEEE Trans. Evol. Comput., vol. 16, no. 1, pp. 117-134, Feb. 2012.

17. E. Mezura-Montes and C. A. C. Coello, "A simple multimembered evolution strategy to solve constrained optimization problems", IEEE Trans. Evol. Comput., vol. 9, no. 1, pp. 1-17, Feb. 2005.

18. K. Weeber and S. R. H. Hoole, "Geometric parametrization and constrained optimization techniques in the design of salient pole synchronous machines", IEEE Trans. Magn., vol. 28, no. 4, pp. 19481960, Jul. 1992.

19. X. Liu and G. R. Slemon, "An improved method of optimization for electrical machines", IEEE Trans. Energy Convers., vol. 6, no. 3, pp. 492-496, Sep. 1991.

20. A. Fatemi, D. M. Ionel, N. A. O. Demerdash and T. W. Nehl, "Fast multi-objective CMODE-type optimization of electric machines for multicore desktop computers", Proc. IEEE Energy Convers. Congr. Expo. (ECCE), pp. 5593-5600, 2015.

21. T. J. E. Miller and J. R. Hendershot, Design of Brushless Permanent-Magnet Machines, Venice, FL, USA:Motor Design Books, 2010.

22. E. C. Lovelace, T. M. Jahns, T. A. Keim and J. H. Lang, "Mechanical design considerations for conventionally laminated high-speed interior PM synchronous machine rotors", IEEE Trans. Ind. Appl., vol. 40, no. 3, pp. 806-812, May/Jun. 2004.

23. D. M. Ionel et al., "Computation of core losses in electrical machines using improved models for laminated steel", IEEE Trans. Ind. Appl., vol. 43, no. 6, pp. 1554-1564, Nov./Dec. 2007.

24. P. Zhang, G. Y. Sizov, J. He, D. M. Ionel and N. A. O. Demerdash, "Calculation of magnet losses in concentrated-winding permanent-magnet synchronous machines using a computationally efficient finite-element method", IEEE Trans. Ind. Appl., vol. 49, no. 6, pp. 2524-2532, Nov./Dec. 2013.

25. E. Zitzler and L. Thiele, "Multiobjective evolutionary algorithms: A comparative case study and the strength Pareto approach", IEEE Trans. Evol. Comput., vol. 3, no. 4, pp. 257-271, Nov. 1999.

26. C. M. Fonseca, P. J. Fleming, E. Zitzler, L. Thiele and K. Deb, "The measure of Pareto optima: Applications to multiobjective metaheuristics", Proc. 2nd Int. Evol. Multi Criterion Optim. (EMO), pp. 519-533, 2003. 